\title{
Role of the superior turbinate when performing endoscopic endonasal transsphenoidal approach
}

\author{
J.-H. Shin ${ }^{1}$, S.G. Kang ${ }^{2}$, Y.K. Hong ${ }^{2}$, S.S. Jeun², S.W. Kim¹, S.W. Kim¹, J.H. Cho1, Y.J. Park ${ }^{1}$ \\ ${ }^{1}$ Department of Otolaryngology — Head and Neck Surgery, The Catholic University of Korea, \\ College of Medicine, Seoul, Korea \\ 2Department of Neurosurgery, The Catholic University of Korea, College of Medicine, Seoul, Korea
}

[Received 1 August 2013; Accepted 31 August 2013]

Background: This study examined the relationship between the superior turbinate and natural ostium of the sphenoid sinus, as seen during the endoscopic endonasal transsphenoidal approach (EETSA) for sellar lesions and described how to enter the sphenoethmoid cell safely for complete exposure of the sellar floor, including adjacent vital structures such as the prominence of the optic nerve and carotid artery.

Materials and methods: This study retrospectively reviewed the medical records and operative findings of 154 patients, who underwent EETSA between February 2009 and February 2011. We evaluated the location of the natural ostium of the sphenoid sinus relative to the superior turbinate and revealed the clinical significance of the superior turbinate as a surgical guide to enter into the sphenoethmoid cell during EETSA.

Results: The natural ostium of the sphenoid sinus was located medially to the posteroinferior end of the superior turbinate in 151 (98\%) patients. In 1 patient, the natural ostia of the sphenoid sinus were located lateral to the superior turbinate bilaterally. Sphenoethmoid cell was encountered in 53 (34\%) patients. We could easily enter the sphenoethmoid cell at the point where the superior turbinate was attached to the anterior wall of the sphenoid sinus.

Conclusions: The superior turbinate is a good surgical landmark for identifying the natural ostium of the sphenoid sinus and as a guide for the surgical entrance to the sphenoethmoid cell extending to the sphenoid sinus during EETSA. (Folia Morphol 2014; 73, 1: 73-78)

Key words: superior turbinate, sphenoid sinus, natural ostium, transsphenoidal approach, endonasal approach, sellar lesions, sphenoethmoid cell

\section{INTRODUCTION}

The endoscopic endonasal transsphenoidal approach (EETSA) is gradually being adopted for treating lesions of the anterior skull base, including the sellar area. This technique has several advantages, such as minimal invasiveness, direct approach, adequate surgical field, and rapid postoperative recovery $[4,6,7,9,22]$. Despite these advantages, pitfalls include the potential for major injuries to vital structures such as the optic nerve and internal carotid artery [3, 4, 6, 8, 22]. Adequate exposure of the sphenoid sinus cavity is needed so that vital structures in the lateral wall of the sphenoid sinus can be identified. This is necessary for a safe approach

Address for correspondence: S.W. Kim, MD, PhD, Department of Otolaryngology - Head and Neck Surgery, The Catholic University of Korea, 505 Banpo-dong, Seocho-gu, Seoul 137-701, Korea, tel: +82 22258 6216, fax: +82 2595 1354, e-mail: kswent@catholic.ac.kr 
to sellar lesions and for a more complete resection of pituitary tumour.

This article reports the relationship of the superior turbinate and natural ostium of the sphenoid sinus as found during the EETSA. We also discuss how the superior turbinate can be used as a surgical landmark for safe entrance into the sphenoethmoid cell to expose the entire sellar floor in cases with a sphenoethmoid cell during the EETSA.

\section{MATERIALS AND METHODS}

The study was performed at the tertiary university hospital. We performed a retrospective review of medical charts, including the operative records, for procedures carried out between February 2009 and February 2011. In total, 154 patients underwent removal of an anterior skull-base tumour, including pituitary lesions, using EETSA.

Preoperatively, we carefully reviewed the paranasal sinus computed tomography (PNS-CT), including axial, coronal, and sagittal views, for all the patients. PNS-CT was obtained in the axial projection with the administration of intravenous contrast material with the patients in the supine position. The PNS-CT images were acquired at $120 \mathrm{kV}$ and $180 \mathrm{~mA}$, with a $7 \mathrm{~s} \mathrm{scan}$ time. The PNS-CT images included $0.6 \mathrm{~mm}$ axial images and coronal and sagittal images reconstructed in $3 \mathrm{~mm}$ slices. All images were examined with a bone (window $2000 \mathrm{HU}$, level $400 \mathrm{HU}$ ) shadow. In particular, we checked the anatomic variants of the sphenoid sinus, such as septation and pneumatisation of the sphenoid sinus and the presence and shape of any sphenoethmoid cell. This work was approved by the institutional review board of Seoul St. Mary's Hospital, The Catholic University of Korea.

\section{Surgical procedures}

Each EETSA was performed by a team that included a rhinologist and a neurosurgeon, and the entire procedure was performed via an endonasal approach with the endoscope. General anaesthesia was induced, and the nasal cavity was decongested with a 1:100,000 epinephrine-soaked cotton pledget. Before the surgery, the nasal septum around the choana and posterolateral and anterolateral attachments of the middle turbinate was injected bilaterally with $1 \%$ lidocaine with epinephrine diluted 1:100,000. We also injected the entire area of the right nasal septum and posterior portion of the left nasal septum to reduce any bleeding and to facilitate hydrodissection of the subperichondrial and subperiosteal layers for preparation of pedicled septal mucosal flap. The bilateral inferior turbinates were out-fractured to enhance manipulation of the endoscope and instruments, and then both middle turbinates were gently and fully lateralised to allow visualisation of the superior turbinate. In most cases, we could easily find the natural ostium of the sphenoid sinus medially to the superior turbinate after lateralising the superior turbinate. In cases with an anticipated large defect of the anterior skull base and cerebrospinal fluid leakage after the surgery, we harvested a pedicled septal mucosal flap for reconstructing the anterior skull base. After elevating the right pedicled septal mucosal flap, we performed a posterior septectomy that included a portion of the perpendicular plate of the ethmoid bone, the vomer, and the anterior wall of the sphenoid sinus. During that surgical procedure, we tried to remove bony material for reconstructing the sellar floor en bloc. After posterior septectomy, we could find the sellar floor. Then, we removed the remnant left posterior septal mucosa for binostril and 4-hand approach to the anterior skull base lesions. Mucosal bleeding was controlled with 1:100,000 epinephrine-soaked cotton pledgets and Surgicel ${ }^{\circledR}$ (Ethicon, Somerville, NJ), and major bleeding was controlled with suction electrocautery.

In the patients with a sphenoethmoid cell, we first removed the remnant mucosa of the anterior wall of the sphenoid sinus around the attachment site of the superior turbinate using a powered instrument. Then, we opened the sphenoethmoid cell using an antrum sicker or curved curette in an oblique anterior-posterior direction at the point, where the superior turbinate was attached to the anterior wall of the sphenoid sinus. An incision along the oblique attachment of the superior turbinate to the anterior wall of the sphenoid sinus made with a sharp tipped electrocautery facilitated the entry into the sphenoethmoid cell with curved instruments. After entering the sphenoethmoid cell, we removed the posterior ethmoidal bony wall extending to the sphenoid sinus with cutting forceps and a powered instrument. This allowed us to expose the entire sellar floor and adjacent vital structures, such as the optico-carotid recess.

\section{Statistical analysis}

We have analysed only the location of the natural ostium of the sphenoid sinus with independent T-test using SPSS version 12 (SPSS Inc., Chicago, IL, USA). A p value $<0.05$ was accepted as statistically significant. 


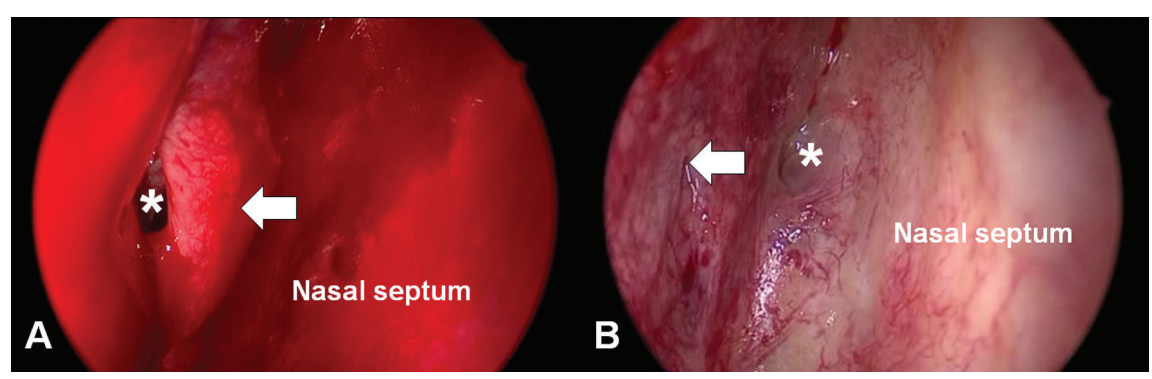

Figure 1. Identification of the natural ostium at the anterior wall of the sphenoid sinus in the right nasal cavity with 0-degree nasal endoscope; A. The natural ostium (asterisk) drains laterally to the postero-inferior portion of the superior turbinate (white arrow); B. The natural ostium (asterisk) of the sphenoid sinus is located medial to the superior turbinate (white arrow) with swollen covering mucosa.

\section{RESULTS}

The study included 154 patients undergoing anterior skull base tumour removal via EETSA: 88 (57\%) males, 66 (43\%) females; mean age 47 (range 10-84) years. The natural ostium of the sphenoid sinus was located medially to the portion of attachment in all but 3 patients $(p=0.000)$ : in 1 , there were bilateral natural ostia lateral to the superior turbinate, and in the other 2, they were located laterally in the right nasal cavity. The posteroinferior end of the superior turbinate was a better reference point for locating the natural ostium of the sphenoid sinus (Fig. 1).

A sphenoethmoid cell was found in 53 (34\%) of 154 patients when performing EETSA. We could enter the sphenoethmoid air cell at the point between the remnant mucosa of the sphenoid sinus anterior wall and lateral attachment of the superior turbinate relatively easily and thereby expose the entire sellar floor and surrounding vital structures, such as the optico-carotid recess (Fig. 2). No immediate complications or injuries to the surrounding vital structures were seen.

\section{DISCUSSION}

The sphenoid sinus is deep in the center of the cranial cavity, surrounded by several important anatomic structures, including the internal carotid artery and the optic nerve. There is much variation in its growth and pneumatisation. Endoscopic surgery of sphenoid sinus and sellar lesions requires clear identification of the sinus anatomy and proper orientation within the surgical cavity to avoid complications, such as a cerebrospinal fluid leak, major intracranial penetration, optic nerve trauma, or major bleeding caused by an internal carotid artery injury. In determining the orientation of the sphenoid sinus, the sphenoid sinus ostium is an important landmark for the anterior sphenoid wall and sellar floor. Several reports have referred to the superior turbinate as a reliable anatomical landmark of the sphenoid sinus and its natural ostium $[2,13,15,16,18]$. Most reports state that the natural ostium of the sphenoid sinus is positioned medial to the superior turbinate $[2,16]$.

The natural ostium of the sphenoid sinus can be difficult to identify during the endoscopic sinus surgery. Successful identification assures the surgeon of the location of the sphenoid sinus, and a number of methods have been proposed to localise the sphenoid sinus accurately. As a traditional technique for identifying the natural ostium of the sphenoid sinus from the anterior aspect of the nose, distances and angles were measured principally from and relative to the anterior nasal spine [5, 20]. Turgut et al. [20] reported that the natural ostium is located approximately $80 \mathrm{~mm}$ from the anterior nasal spine, and Davis et al. [5] reported its location to be $30^{\circ}$ and $7 \mathrm{~cm}$ relative to the horizontal plane of the nasal spine. Using the subnasale as a reference point, Lang [11] described it as being $62 \mathrm{~mm}$ away. Kim et al. [10] showed that it was $6.3 \mathrm{~cm}$ from the sill and that the angle was approximately $34^{\circ}$.

Surgically, the location of the natural ostium of the sphenoid sinus has been reported using different reference points. It is $10-20 \mathrm{~mm}$ superior to the base of the sphenoid sinus $4-3 \mathrm{~mm}$ medially and $5 \mathrm{~mm}$ superior to the posterior end of the inferior turbinate; it is located 10-12 mm superior to the top of the choana [21]. Levin and May [13] suggested that it is much easier to find the sphenoid sinus when using the posteroinferior end of the middle turbinate as a reference point.

Several reports refer to the superior turbinate as a landmark for the sphenoid natural ostium. Orlandi et al. [18] reported on the use of the superior turbinate as a constant, reliable landmark for the natural ostium of the sphenoid sinus. When the posterior por- 


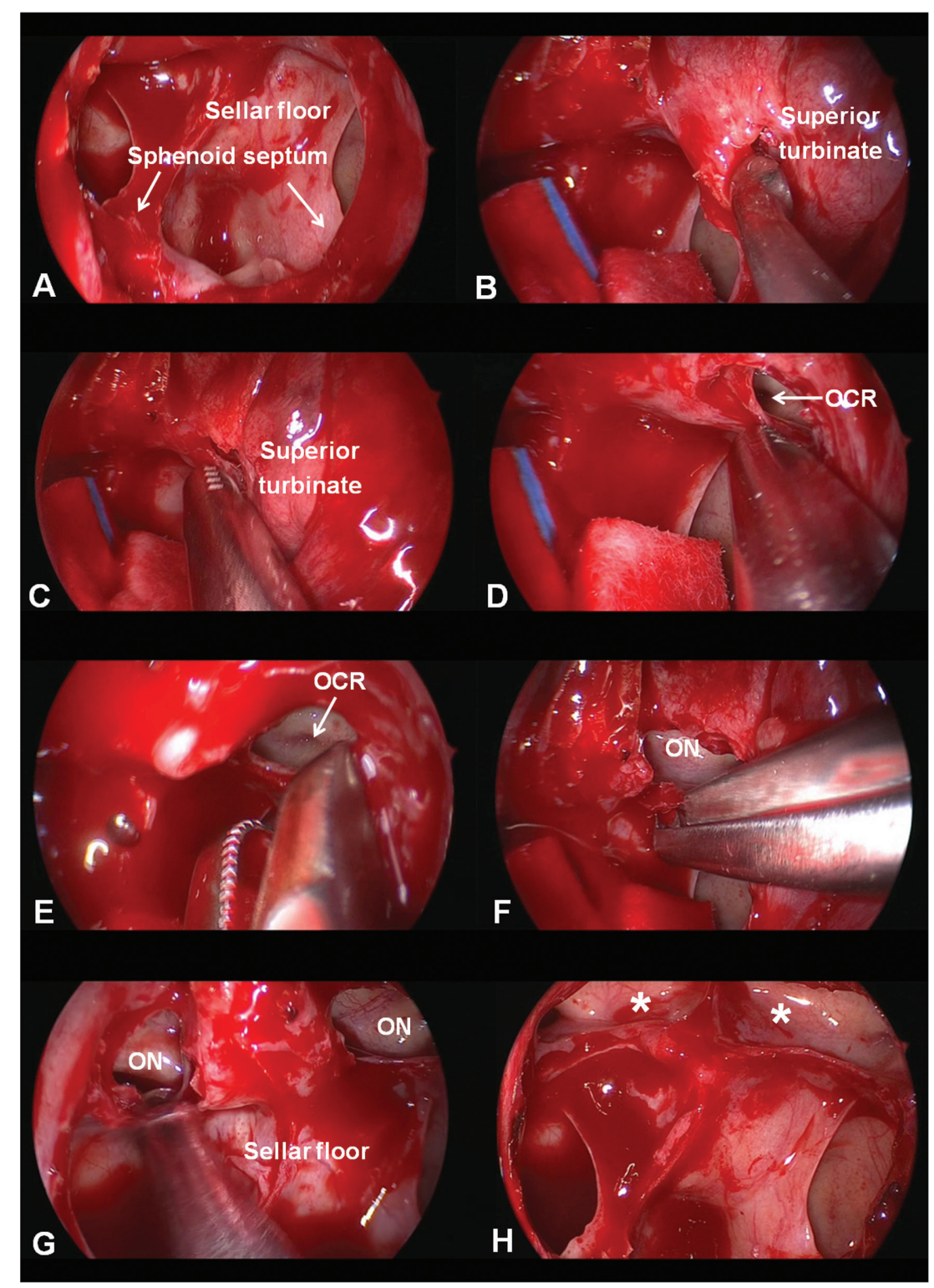

Figure 2. A. The findings with a 0-degree nasal endoscope after a partial septectomy with removal of the anterior wall of the sphenoid sinus. The lower half of the sellar floor is seen before the entrance of sphenoethmoid cell; B. Opening of the left sphenoethmoid cell using a 45-degree curved curette at the point where the superior turbinate is attached to the anterior wall of the sphenoid sinus in an oblique anterior-posterior direction; C, D. After entering the left sphenoethmoid cell, the posterior ethmoidal bony wall between the sphenoid sinus and posterior ethmoid cell is removed with a powered instrument; E, F. The remaining posterior ethmoidal bony wall is removed with upward cutting forceps and a rongeur, and the left optico-carotid recess (OCR) and optic nerve $(\mathrm{ON})$ are seen through a 30-degree nasal endoscope; G. The right sphenoethmoid cell is opened with a powered instrument; $\mathbf{H}$. This procedure allowed us to expose the entire sellar floor and adjacent vital structures such as the optico-carotid recess. Asterisk indicates the sphenoethmoid cell.

tion of the superior turbinate is resected, it gives wide exposure to the sphenoid sinus natural ostium. Kim et al. [10] analysed 100 cadaveric specimens that had been sectioned in the sagittal plane and found that the sphenoid sinus ostium was medial to the superior turbinate in 54 of $65(83.1 \%)$ specimens. Millar and Orlandi [15] reported that the natural ostium of the sphenoid sinus was medial to the superior turbinate in all of their 47 cadaveric specimens. Min et al. [16] and
Bolger et al. [2] suggested that the superior meatus and superior turbinate were reliable landmarks for the sphenoid sinus in the transsphenoidal approach. In our study, the trans-superior meatal approach was performed in 154 patients with sellar lesions. In most cases, we have found that the natural ostium was medial to the superior turbinate, as expected. After considering the relationships between the natural ostium of the sphenoid sinus and the superior 
turbinate, we entered through the medial portion of the superior turbinate to find the natural ostium of the sphenoid sinus. In 3 patients, however, we have found that the natural ostium of the sphenoid sinus was located lateral to the site of attachment. In such cases, we found the natural ostium after medialising the superior turbinate.

The posterior expansion of the posterior ethmoid cell over the sphenoid sinus in particular leads to errors in determining the orientation of the sphenoid sinus. There is a major risk of adverse events when there is poor orientation and a blind operation is performed. There is marked projection of the posterior ethmoid cells over and into the sphenoid sinus in $8.4-25 \%$ of the cases $[1,12,14,17,19]$. This often makes sphenoid sinus identification even more difficult and at times dangerous. In patients with a sphenoethmoid cell, its removal enables complete exposure of the sellar lesion and allows the surgeon to identify the vital structures. Incomplete exposure of the sphenoid sinus and related vital structures can lead to severe complications.

As a fourth basal lamella, the superior turbinate is the structure between the posterior ethmoid and the anterior wall of the sphenoid sinus. It is located immediately medial to the posterior ethmoid cavity, with its leading edge just posterior to the middle turbinate in the same parasagittal plane. Consequently, the posterior mucosa of the superior turbinate joins the anterior wall of the sphenoid sinus in an oblique direction. It is the same in patients with a sphenoethmoid cell. Therefore, the superior turbinate would be a useful structure for distinguishing the posterior-most ethmoid cell from the sphenoid sinus [13]. On this anatomical basis, we used the superior turbinate as a guide for the surgical entrance to the sphenoethmoid air cell in the sphenoid sinus with the EETSA. We opened the sphenoethmoidal air cell using an antrum seeker or J-curette in an oblique anterior-posterior direction at the point where the superior turbinate was attached to the anterior wall of the sphenoid sinus. In this manner, we exposed the entire sellar area and adjacent vital structures. Consequently, our surgical technique using the superior turbinate would be a good model for opening the sphenoethmoid cell.

\section{CONCLUSIONS}

The superior turbinate is a good surgical landmark for identifying the natural ostium of the sphenoid sinus in the EETSA. The entire superior turbinate was lateralised fully without resecting this anatomical structure, giving wide exposure to the sphenoid sinus natural ostium in most cases. Another important role of the superior turbinate is a guide for the surgical entrance to the sphenoethmoid cell extending into the sphenoid sinus. The posterior mucosa of the superior turbinate joins the anterior wall of the sphenoid sinus in an oblique direction. At this junction, we could easily enter into the posterior ethmoid sinus in cases with sphenoethmoid cell.

\section{ACKNOWLEDGEMENTS}

This research was supported by Seoul St. Mary's Clinical Medical Research Program year of 2013 through the Catholic University of Korea.

\section{REFERENCES}

1. Akdemir G, Tekdemir I, Altin L (2004) Transethmoidal approach to the optic canal: Surgical and radiological microanatomy. Surg Neurol, 62: 263-274.

2. Bolger WE, Keyes AS, Lanza DC (1999) Use of the superior meatus and superior turbinate in the endoscopic approach to the sphenoid sinus. Otolaryngol Head Neck Surg, 120: 308-313.

3. Cappavianca P, Cavallo LM, Colao A, de Divitiis (2002) Surgical complications associated with the endoscopic endonasal transsphenoidal approach for pituitary adenomas. J Neurosurg, 97: 293-298.

4. Ceylan S, Koc K, Anik I (2010) Endoscopic endonasal transsphenoidal approach for pituitary adenomas invading the cavernous sinus. J Neurosurg, 112: 99-107.

5. Davis WE, Templer J, Parsons DS (1996) Anatomy of the paranasal sinuses. Otolaryngol Clin North Am, 29: 57-74.

6. de Divitiis E, Cavallo LM, Cappabianca P, Esposito F (2007) Extended endoscopic endonasal transsphenoidal approach for the removal of suprasellar tumors. Part 2. Neurosurgery, 60: 46-58.

7. Dehdashti AR, Ganna A, Karabatsou K, Gentili F (2008) Pure endoscopic endonasal approach for pituitary adenomas : early surgical results in 200 patients and comparison with previous microsurgical series. Neurosurgery, 62: 1006-1015.

8. Frank G, Pasquini E, Farneti G, Mazzatenta D, Sciarretta V, Grasso V, Faustini M (2006) The endoscopic versus the traditional approach in pituitary surgery. Neuroendocrinology, 83: 240-248.

9. Hackson A, Joao Paulo C, Gomes E (2010) Endoscopic endonasal transsphenoidal surgery: surgical results of 228 pituitary adenomas treated in a pituitary center. Pituitary, 13: 68-77.

10. Kim HU, Kim SS, Kang SS, Chung IH, Lee JG, Yoon JH (2001) Surgical anatomy of the natural ostium of the sphenoid sinus. Laryngoscope, 111: 1599-1602.

11. Lang J (1989) Clinical anatomy of the nose, nasal cavity and paranasal sinuses. Thieme Medical, New York, pp. 85-98. 
12. Leunig A, Betz CS, Sommer B, Sommer F (2008) Anatomic variations of the sinuses; multiplanar CT-analysis in 641 patients. Laryngorhinootologie, 87: 482-489.

13. Levin HL, May M (1993) Endoscopic sinus surgery. Thieme Medical, New York, pp. 142-144.

14. Mazza D, Bontempi E, Guerrisi A, Del Monte S, Cipolla G, Perrone A, Lo Mele L, Marini M (2007) Paranasal sinuses anatomic variants: 64 -slice CT evaluation. Minerva Stomatol 56: 311-318.

15. Millar DA, Orlandi RR (2006) The sphenoid sinus natural ostium is consistently medial to the superior turbinate. Am J Rhinol, 20: 180-181.

16. Min YG, Shin JS, Lee CH (1995) Trans-superior meatal approach to the sphenoid sinus. ORL J, 57: 289-292.

17. Nitinavakarn B, Thanaviratananich S, Sangsilp N (2005) Anatomical variations of the lateral nasal wall and paranasal sinuses: a CT study for endoscopic sinus surgery (ESS) in Thai patients. J Med Assoc Thai, 88: 763-768.
18. Orlandi RR, Lanza DC, Bolger WE, Clerico DM, Kennedy DW (1999) The forgotten turbinate: The role of the superior turbinate in endoscopic sinus surgery. Am J Rhinol, 13: 251-259.

19. Tan HK, Ong YK (2007) Sphenoid sinus: an anatomic and endoscopic study in Asian cadavers. Clin Anat, 20: 745-750.

20. Turgut S, Gumusalan Y, Arifoglu Y, Sinav A (1996) Endoscopic anatomic distances on the lateral nasal wall. J Otolaryngol, 25: 371-374.

21. Wigand ME (1990) Endoscopic surgery of the paranasal sinuses and anterior skull base. Thieme Medical, New York, pp. 53-56.

22. Zhao B, Wei YK, Li GL, Li YN, Yao Y, Kang J, Ma WB, Yang Y (2010) Extended transsphenoidal approach for pituitary adenomas invading the anterior cranian base, cavernous sinus, and clivus: a single-center experience with 126 consecutive cases. J Neurosurg, 112: 108-117.. 